\title{
Saturated and Unsaturated Dietary Fats Differentially Modulate Ethanol-Induced Changes in Gut Microbiome and Metabolome in a Mouse Model of Alcoholic Liver Disease
}

Irina A. Kirpich, ${ }^{\star \dagger}$ Joseph Petrosino, ${ }^{\ddagger \S}$ Nadim Ajami, ${ }^{\ddagger \S}$ Wenke Feng, ${ }^{* \dagger}$ Yuhua Wang, ${ }^{* \dagger}$ Yanlong Liu, ${ }^{* \dagger \|}$ Juliane I. Beier, Shirish S. Barve, ${ }^{* \dagger}$ Xinmin Yin, ${ }^{* *}$ Xiaoli Wei, ${ }^{* *}$ Xiang Zhang, ${ }^{\dagger * *}$ and Craig J. McClain ${ }^{* \dagger, \dagger \dagger}$

From the Division of Gastroenterology, Hepatology, and Nutrition, * Department of Medicine, and the Departments of Pharmacology and Toxicology ${ }^{\dagger}$ and Chemistry, ** University of Louisville School of Medicine, Louisville, Kentucky; the Baylor Department of Molecular Virology and Microbiology $\ddagger$ and the Alkek Center for Metagenomics and Microbiome Research, ${ }^{\S}$ Baylor College of Medicine, Houston, Texas; the College of Food Science and Engineering, ${ }^{\circledR}$ Jilin Agricultural University, Changchun, China; the School of Pharmaceutical Sciences," Wenzhou Medical University, Wenzhou, China; and the Robley Rex Veterans Medical Center, ${ }^{\dagger \dagger}$ Louisville, Kentucky

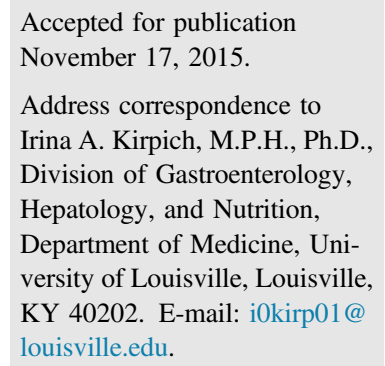

\begin{abstract}
Alcoholic liver disease (ALD) ranks among major causes of morbidity and mortality. Diet and crosstalk between the gut and liver are important determinants of ALD. We evaluated the effects of different types of dietary fat and ethanol on the gut microbiota composition and metabolic activity and the effect of these changes on liver injury in ALD. Compared with ethanol and a saturated fat diet (medium chain triglycerides enriched), an unsaturated fat diet (corn oil enriched) exacerbated ethanol-induced endotoxemia, liver steatosis, and injury. Major alterations in gut microbiota, including a reduction in Bacteroidetes and an increase in Proteobacteria and Actinobacteria, were seen in animals fed an unsaturated fat diet and ethanol but not a saturated fat diet and ethanol. Compared with a saturated fat diet and ethanol, an unsaturated fat diet and ethanol caused major fecal metabolomic changes. Moreover, a decrease in certain fecal amino acids was noted in both alcohol-fed groups. These data support an important role of dietary lipids in ALD pathogenesis and provide insight into mechanisms of ALD development. A diet enriched in unsaturated fats enhanced alcohol-induced liver injury and caused major fecal metagenomic and metabolomic changes that may play an etiologic role in observed liver injury. Dietary lipids can potentially serve as inexpensive interventions for the prevention and treatment of ALD. (Am J Pathol 2016, 186: 765-776; http://dx.doi.org/10.1016/j.ajpath.2015.11.017)
\end{abstract}

Alcoholic liver disease (ALD) ranks among major causes of morbidity and mortality in the United States and worldwide. ALD includes a spectrum of conditions from simple steatosis to alcoholic steatohepatitis characterized by inflammation, with potential progression to fibrosis and cirrhosis over time. Alcoholic hepatitis occurs in approximately $10 \%$ to $35 \%$ of chronic drinkers, and severe alcoholic hepatitis accounts for significant morbidity and mortality approaching $35 \%$ to $45 \%$. $^{1}$ Approximately, $10 \%$ to $20 \%$ of heavy drinkers will develop cirrhosis. ${ }^{2,3}$

The crosstalk between the gut and liver is an important determinant of alcohol-induced liver disease. ${ }^{4-6}$ Several studies have found that alcohol consumption (both acute and chronic) increases bacteria-derived products, specifically lipopolysaccharide (LPS) and bacterial DNA, in the portal and systemic circulation. ${ }^{7,8}$ Significantly increased levels of LPS, which induces a proinflammatory response in the liver, were found in patients with different stages of ALD (fatty liver, hepatitis, and cirrhosis) ${ }^{9,10}$ and in experimental animal models of ALD. ${ }^{1-13}$ As found in a

\footnotetext{
Supported by NIH grants R21 AA020849-01A1 (I.A.K.), U01AA022489, 1U01AA021901-01, 1U01AA021893-01, R01AA023681, and R01AA018869 (C.J.M.), the Department of Veterans Affairs grant BX000350 (C.J.M.), and Department of Defense grant W81XWH-11-10595 (C.J.M.).

Disclosures: None declared.
} 
number of recent clinical $^{14-18}$ and preclinical studies, ${ }^{12,19,20}$ alcohol intake and alcohol-induced liver injury are associated with qualitative (dysbiosis) and quantitative (bacterial overgrowth) alterations of gut microbiota. The molecular mechanisms by which the altered gut microbiota contribute to ALD are not well understood. Alcohol-mediated changes in the gut microbiota facilitate disruption of gut barrier integrity, ${ }^{12,19,21}$ resulting in increased intestinal permeability to bacteria-derived products, which eventually contributes to the development of ALD. Bacteria overgrowth may enhance ethanol production and metabolism with subsequent high concentrations of acetaldehyde ${ }^{22}$ (product of ethanol degradation), which is known to affect intestinal intercellular junctions. ${ }^{23}$ Mice that were protected from intestinal bacterial overgrowth and dysbiosis had decreased alcohol-induced endotoxemia and liver disease. ${ }^{24}$ Antibiotic treatment to sterilize the gut, ${ }^{25,26}$ as well as prebiotics or probiotics administration to normalize the gut microbiota community, ${ }^{19,27,28}$ attenuates alcohol-induced intestinal barrier leakage and decreases endotoxemia and hepatic injury in rodents. The link between the dietary fat and alcohol in ALD is increasingly recognized. Several studies, including those from our laboratory, have found that dietary unsaturated fat exacerbates alcohol-mediated intestinal permeability, liver steatosis, inflammation, and injury. ${ }^{11,29-32}$ These pathologic effects were prevented or blunted by dietary saturated fat, suggesting a significant contribution of specific dietary lipids in ALD development and progression. However, the exact mechanism(s) underlying these effects remains to be established. In the present study, we evaluated the response of the gut microbiota (in terms of composition and metabolic activity) to ethanol and different types of dietary fat in an experimental animal model of ALD. The results of the study contribute to understanding the complexity of the interplay among the diet, gut microbiota, and ethanol-induced fatty liver disease.

\section{Materials and Methods}

\section{Animals and Treatments}

C57BL/6N male mice obtained from Harlan Laboratories, Inc. (now Envigo RMS, Indianapolis, IN) were pair-fed control or ethanol-containing (5\% ethanol v/v) diets ad libitum for 8 weeks (Figure 1A). ${ }^{11}$ Mice were fed a modified LieberDeCarli liquid diet (Research Diet, New Brunswick, NJ) that contained saturated fat (SF) or unsaturated fat (USF). The SF or USF diets were enriched in medium chain triglycerides (MCTs) and beef tallow (18:82 ratio) or corn oil, respectively (Figure 1B). The detailed dietary fatty acid composition has been described previously. ${ }^{32}$ Control mice were pair-fed SF or USF maltose-dextrin diets that were isocaloric with the ethanol diets. In the control group diets, the levels of protein, carbohydrate, and fat were held constant at $17 \%, 43 \%$, and $40 \%$ of total energy, respectively. In the alcohol diets, ethanol (35\% of total calories) was substituted for carbohydrate energy. At the
A EtOH fed
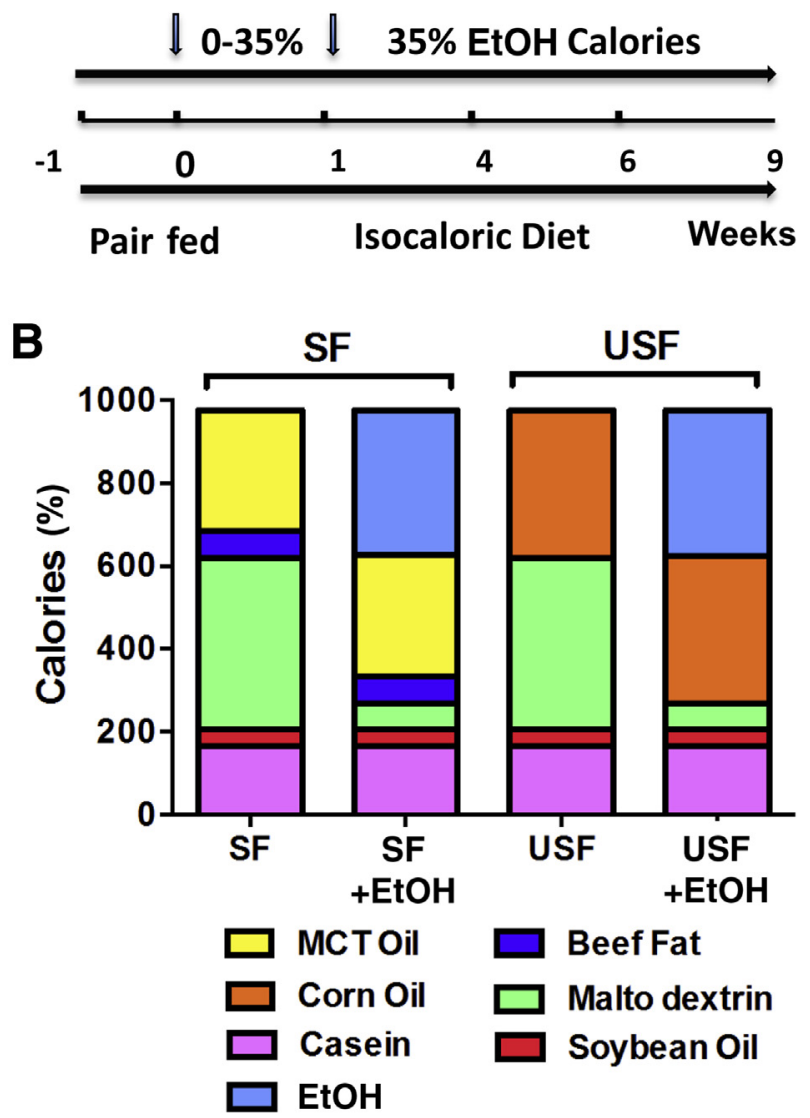

Figure 1 The experimental animal model of alcoholic liver disease. A: The schematic presentation of chronic ethanol $(\mathrm{EtOH})$ or control diet feeding protocol. Initially, all mice were given the control liquid maltose dextrin diets [saturated fat (SF) or unsaturated fat (USF), no ethanol] ad libitum for 1 week. Afterward, mice were fed either the liquid ethanolcontaining diets or the control liquid maltose-dextrin diets. Ethanol was gradually increased to $35 \%$ of total calories $(5.0 \% \mathrm{v} / \mathrm{v})$. The mice were fed the ethanol diet ( $5 \%$ ethanol $v / v)$ ad libitum for 8 weeks. B: The composition of the experimental liquid diets. The SF diet was enriched with medium chain triglyceride oil and beef tallow fat (82:18 ratio). The USF diet was enriched with corn oil. Soybean oil was used in both diets to provide essential free fatty acids. The control (SF and USF) diets contained $43 \%$ of calories from carbohydrate, $17 \%$ from protein, and $40 \%$ from fat. The SF and ethanol and USF and ethanol diets contained 35\% of calories from ethanol to replace the calories from carbohydrate.

end of the study period, the mice were anesthetized, and blood, liver, and intestinal tissue samples were collected. Stool samples were collected weekly throughout the study. Animals were housed in a pathogen-free barrier facility accredited by the Association for Assessment and Accreditation of Laboratory Animal Care, and the study protocol was approved by the University of Louisville Institutional Animal Care and Use Committee.

\section{Blood and Liver Biochemical Analysis}

Plasma endotoxin levels were measured with the limulus amebocyte lysate kit (Lonza, Walkersville, MD) according to 
the manufacturer's instructions. Plasma alanine aminotransferase and hepatic triglyceride levels were determined as described previously ${ }^{33}$ using commercially available reagents from Thermo Fisher Scientific Inc. (Middletown, VA).

\section{Liver Histologic Examination and Staining}

For histologic analysis, liver sections were fixed in $10 \%$ buffered formalin and embedded in paraffin. Tissue sections $(5 \mu \mathrm{m})$ were prepared and stained with hematoxylin and eosin. Apoptotic cells were identified by terminal deoxynucleotidyl transferase-mediated dUTP nick-end labeling assay using the ApopTag Peroxidase In Situ Apoptosis Detection kit (Millipore, Billerica, MA). Neutrophil accumulation in the livers was assessed by chloracetate esterase staining using a commercially available kit (Sigma-Aldrich, St. Louis, MO). Macrophage infiltration was determined by F4/80 (Abcam, Cambridge, MA) staining.

\section{Fecal Metagenomic Analysis}

The fecal microbial ecosystem was investigated using standard molecular techniques (16S rRNA Gene Sequencing and Analysis) as previously described. ${ }^{19}$ Briefly, bacterial genomic DNA was extracted from fecal samples belonging to mice from each exposure group using the MO BIO Powersoil DNA Extraction Kit (Carlsbad, CA). The V3-V5 16S rRNA gene variable regions were amplified by PCR using 454 adapterlinked and bar-coded primers 357F and 926R, as described previously. ${ }^{34}$ Amplicons were purified using a SPRI bead clean-up step, quantitated by picogreen assay, normalized, pooled, and then sequenced on a 454 instrument using the FLX Titanium chemistry. Filtered data were analyzed using both operational taxonomic unit and taxonomic binning of classified sequences. The Ribosomal Database Project ${ }^{35}$ classifier was used to identify the taxonomic classification of the sequence reads.

\section{Fecal Metabolomic Analysis}

\section{Metabolite Sample Preparation}

The fecal samples were weighed and homogenized after adding ice-cold $80 \%$ methanol at a concentration of $20 \mathrm{mg} / \mathrm{mL}$. The homogenized samples were sonicated for 30 minutes, vortexed for 10 minutes, followed by centrifugation at $4^{\circ} \mathrm{C}$ for 10 minutes at $9000 \times g$. The superna$\operatorname{tant}(650 \mu \mathrm{L})$ was transferred into a plastic tube, which was then dried overnight in a centrifugal evaporator (SpeedVac; Thermo-Fisher, Waltham, MA). The extracted metabolites were then dissolved in a $70-\mu \mathrm{L}$ mixture of acetonitrile and $N$-(tert-butyldimethylsilyl)- $N$-methyltrifluoroacetamide mixed with $1 \%$ tert-butyldimethylchlorosilane. The mixture was sonicated for 1 hour followed by incubation at $80^{\circ} \mathrm{C}$ for 30 minutes. The samples were then transferred to gas chromatography vials for analysis. The derivatization was performed just before comprehensive two-dimensional gas chromatography time-of-flight mass spectrometry $(\mathrm{GC} \times \mathrm{GC}-$ TOF MS) analysis.

\section{$\mathrm{GC} \times \mathrm{GC}-\mathrm{TOF}$ MS Analysis}

The LECO Pegasus 4D GC $\times$ GC-TOF MS instrument (LECO Corp., St Joseph, MI) was equipped with an Agilent 6890 gas chromatograph (Agilent Technologies, Santa Clara, CA) and a Gerstel MPS2 autosampler (Gerstel, Inc., Linthicum, MD), featuring a LECO two-stage cryogenic modulator and secondary oven. The primary column was a $60 \mathrm{~m} \times 0.25 \mathrm{~mm}^{1} d_{c} \times 0.25 \mu \mathrm{m}^{1} d_{f}$, DB-5 ms GC capillary column [phenyl arylene polymer virtually equivalent to (5\%phenyl)-methylpolysiloxane]. A second GC column of $1 \mathrm{~m} \times 0.25 \mathrm{~mm}^{1} d_{c} \times 0.25 \mu \mathrm{m}^{2} d_{f}$, DB-17 ms [ $[50 \%$ phenyl)-methylpolysiloxane] was placed inside the secondary GC oven after the thermal modulator. Both columns were obtained from Agilent Technologies. The helium carrier gas (99.999\% purity) flow rate was set to $2.0 \mathrm{~mL} /$ minute at a corrected constant flow via pressure ramps. The inlet temperature was set at $280^{\circ} \mathrm{C}$. The primary column temperature was programmed with an initial temperature of $60^{\circ} \mathrm{C}$ for 0.5 minutes and then was ramped at $5^{\circ} \mathrm{C}$ per minute to $270^{\circ} \mathrm{C}$ and kept for 15 minutes. The secondary column temperature program was set to an initial temperature of $70^{\circ} \mathrm{C}$ for 0.5 minutes and then ramped at the same temperature gradient used in the first column to $280^{\circ} \mathrm{C}$ accordingly. The thermal modulator was set to $+15^{\circ} \mathrm{C}$ relative to the secondary oven, and a modulation time of $\mathrm{P}_{M}=2$ seconds was used. The mass range was set as 29 to $800 \mathrm{~m} / \mathrm{z}$ with an acquisition rate of 200 mass spectra per second. The ion source chamber was set at $230^{\circ} \mathrm{C}$ with the transfer line temperature set to $280^{\circ} \mathrm{C}$, and the detector voltage was $1450 \mathrm{~V}$ with electron energy of $70 \mathrm{eV}$. The acceleration voltage was turned on after a solvent delay of 674 seconds. The split ratio was set at 25:1.

\section{$\mathrm{GC} \times \mathrm{GC}-\mathrm{TOF}$ MS Data Analysis}

The GC $\times$ GC-TOF MS data were processed using instrument control software ChromaTOF version 4.50.8.0 (LECO Corp.) for peak picking and tentative metabolite identification, followed by retention index matching, peak merging, peak list alignment, normalization, and statistical significance test using MetPP software. ${ }^{36}$ For metabolite identification using ChromaTOF, each chromatographic peak was tentatively assigned to a metabolite if its experimental mass spectrum and a database spectrum have a spectral similarity score of $\geq 600$. The retention index matching in MetPP was performed using the iMatch method with the $P$ value threshold set at $\leq 0.001{ }^{37}$ The pairwise two-tail $t$-test and two-way analysis of variance test (where appropriate) with sample permutation were used to determine whether a metabolite had a statistically significant abundance difference between sample groups.

\section{Statistical Analysis}

Biochemical data are expressed as means \pm SEM. Twoway analysis of variance followed by the Tukey 
A

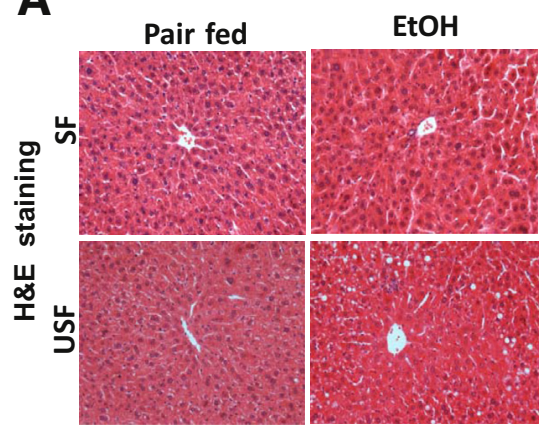

C

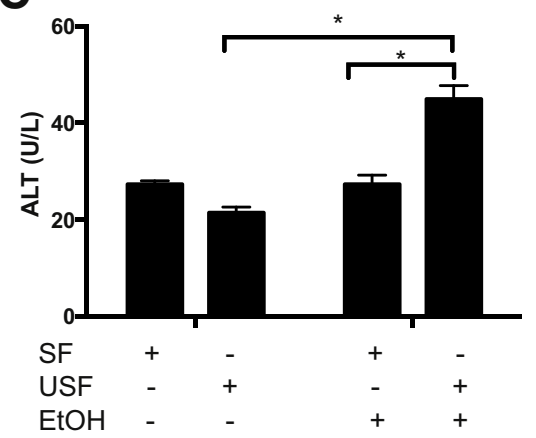

B

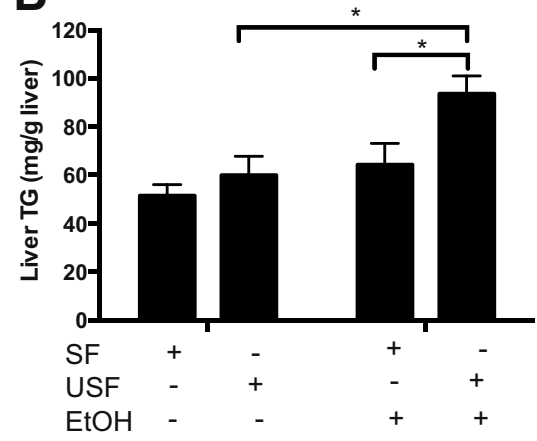

D

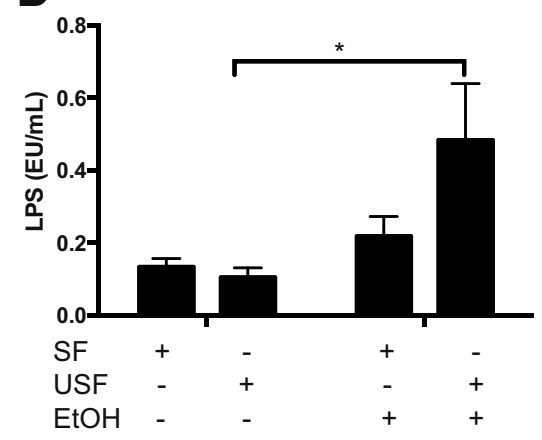

Figure 2 Effects of different dietary fat profile on liver steatosis, injury, and endotoxemia in response to chronic alcohol feeding. A: Representative images of hepatic hematoxylin and eosin (H\&E) staining. B: Liver triglyceride (TG) levels. C: Plasma alanine aminotransferase (ALT) levels. D: Plasma lipopolysaccharide (LPS) levels. Data are expressed as means \pm SEM. $n=6$ to 7 animals per group. ${ }^{*} P<0.05$, two-way analysis of variance, followed by the Tukey multiple-comparison test. Original magnification, $\times 200($ A). EtOH, ethanol; SF, saturated fat; USF, unsaturated fat. multiple-comparison test were used to evaluate differences between the experimental groups. $P<0.05$ was considered statistically significant. Statistical analysis was performed using GraphPad Prism version 5.01 for Windows (GraphPad Software, Inc., La Jolla, CA).

\section{Results}

\section{Characteristics of Liver Disease Caused by Ethanol and Dietary USF}

Compared with SF and ethanol, long-term USF and ethanol feeding resulted in an early stage of ALD characterized by hepatic steatosis (Figure 2, A and B), liver injury with increased plasma ALT levels (Figure 2C), and elevated plasma LPS levels (Figure 2D). USF and ethanol-induced liver steatosis and injury were accompanied by increased hepatic neutrophil (Figure 3, A and B) and macrophage (Figure 3, C and D) infiltration. There were minor differences in hepatic cell apoptosis between animals fed SF and ethanol vs USF and ethanol (Figure 3, $\mathrm{E}$ and $\mathrm{F}$ ). As we have previously reported, intestinal inflammation and increased gut permeability with intestinal tight junction and mucous layer alterations were the characteristics of intestinal impairment caused by USF and EtOH administration in this animal model. ${ }^{11,32}$ Importantly, the pathologic changes that occurred in the liver and the intestine in response to ethanol and dietary USF were not observed in mice fed ethanol and dietary SF, suggesting the differential effects of diverse types of dietary lipids on ethanol-mediated intestinal and liver injury.

Association of Ethanol and Dietary USF-Induced Liver Injury with Gut Microbiota Alterations

The gut microbiota have been increasingly recognized as a critical factor in the ALD development in mice and humans. ${ }^{12,15,19-21,38}$ To investigate whether the observed differences in liver injury between animals fed SF and ethanol and those fed USF and ethanol in our model were associated with the differences in the intestinal microbiota, we performed fecal metagenomic analysis. The 16S rRNA comparative sequence analysis of fecal samples revealed a clear difference in the bacterial composition between animals fed SF and ethanol compared with those fed USF and ethanol with regard to the phylum levels for Bacteroidetes, Actinobacteria, and Proteobacteria (Figure 4A). Specifically, mice fed USF and ethanol compared with those fed SF and ethanol had a notable decrease in Bacteroidetes (approximately sevenfold) without noticeable changes in the abundance of Firmicutes. As a result, the proportional representation of Firmicutes and Bacteroidetes, which is, in general, considered to be of significant relevance in the gut microbiota composition, ${ }^{39}$ was notably altered in mice fed USF and ethanol but not in those fed SF and ethanol (Figure 4B). Notably, neither the SF diet nor the USF diet alone had a significant effect on fecal Firmicutes and Bacteroidetes abundance. Our data suggest that under our experimental conditions a combination of ethanol and 

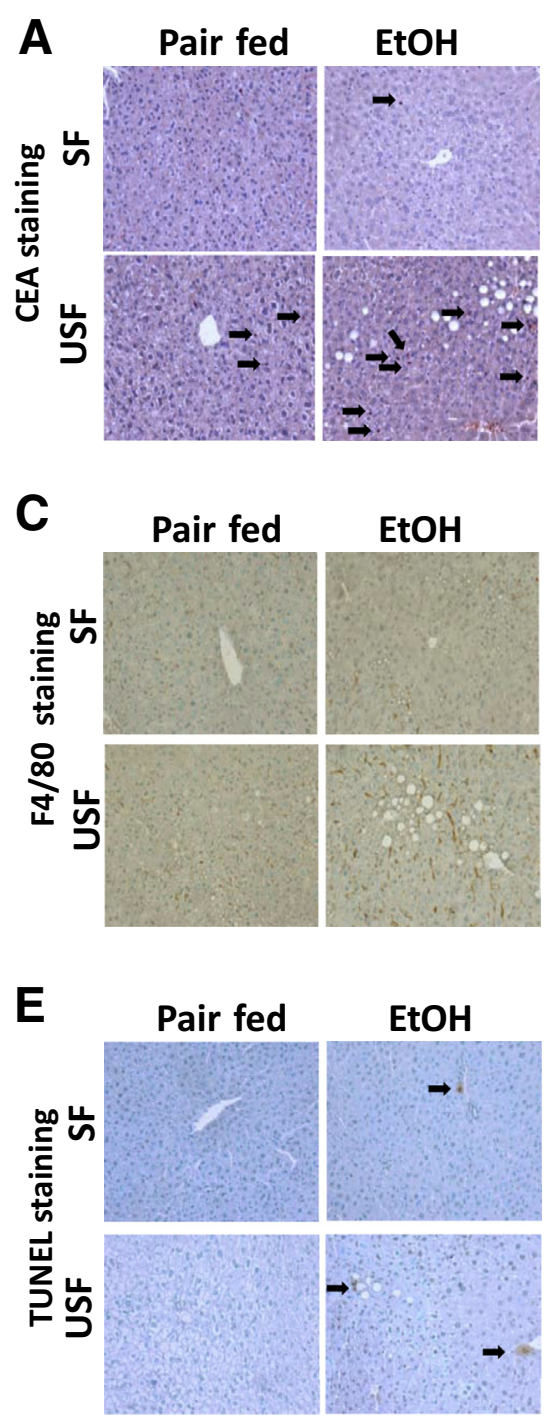

B

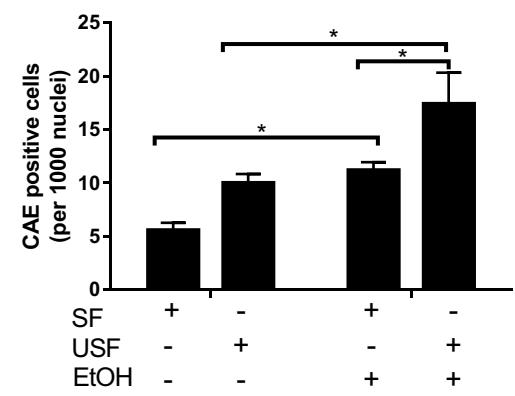

D

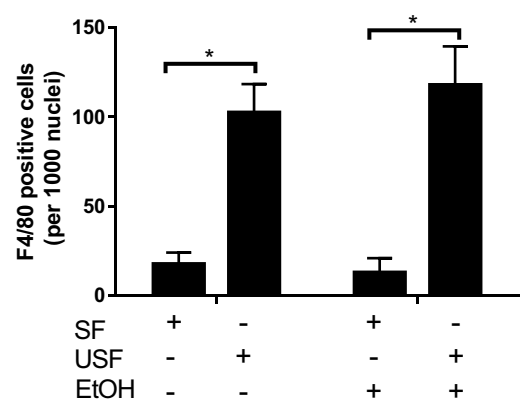

F

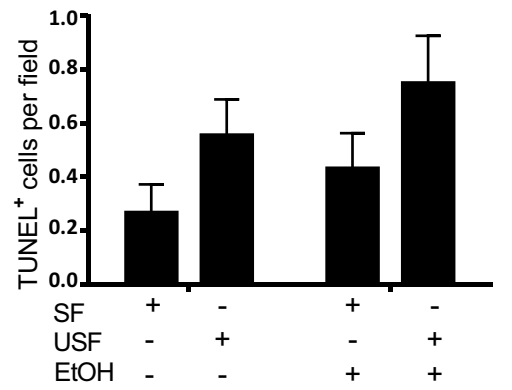

Figure 3 Effects of different types of dietary lipids and chronic ethanol (EtOH) administration on hepatic apoptosis and neutrophil and macrophage infiltration. A and B: Representative images and quantitation of chloroacetate esterase (CAE) staining. Arrows indicate CAE-positive neutrophils. $\mathbf{C}$ and $\mathbf{D}$ : Representative images and quantitation of $F 4 / 80$ staining. The brown staining represents F4/80-positive hepatic macrophages. $\mathbf{E}$ and $\mathbf{F}$ : Representative images of terminal deoxynucleotidyl transferase dUTP nick-end labeling (TUNEL) staining. Arrows indicate TUNEL-positive hepatocytes. Quantitation of representative images was performed by blinded counting of the number of CAE-, F4/80-, and TUNEL-positive cells in random 7 to 10 digital images per animal (I.A.K. and J.I.B.). Data are expressed as means \pm SEM. $n=3$ animals per group. ${ }^{*} P<0.05$. Original magnification: $\times 200($ A, C, and $\mathbf{E})$. SF, saturated fat; USF, unsaturated fat. dietary USF had a major effect on the abundance of Bacteroidetes with limited effects on Firmicutes. Furthermore, in parallel with the decrease in the abundance of Bacteriodetes, USF and ethanol feeding resulted in expansion of the gram-negative Proteobacteria and gram-positive Actinobacteria phyla. These two phyla comprised approximately $40 \%$ of the total bacterial abundance in mice fed USF and ethanol at the end of experimental protocol. Importantly, this major expansion of Proteobacteria and Actinobacteria was not observed in mice in the SF, USF, or SF and ethanol groups. Furthermore, compared with the baseline (the beginning of the experiment), 8 weeks of ethanol administration resulted in a minor increase in Firmicutes in animals fed SF and USF diets, whereas a notable decrease in Bacteroidetes and an increase in Proteobacteria and Actinobacteria occurred only in mice fed a USF and ethanol diet (Figure 4C). Hence, consistent with the recently published studies and extending existing evidence, ${ }^{12,19-21,40}$ our study indicates that the types of dietary fat play a critical role in ethanol-mediated changes of the composition of the gut microbiota.

\section{Effects of Ethanol and SF or USF on the Fecal Metabolome}

To further investigate the potential role of the intestinal microbiota in experimental ALD, we evaluated the gut microbiota metabolic functionality and activity by performing the fecal metabolomic analysis. Metabolites derived from a complex network of microbial metabolic pathways represent chemical characterization of microbiota functional status. Characterization of the function of the microbiota may be more informative and important to the status of host-microbial interaction than the specific microbial composition. In our experiment, the animals were fed diets that contained similar sources and amounts of protein and carbohydrate and different types of dietary fat. The SF diet was enriched in MCTs and beef tallow fat; most free fatty acids in this diet were octanoic (caprylic, $\mathrm{C}_{8} \mathrm{H}_{16} \mathrm{O}_{2}$ ) and decanoic (capric, $\mathrm{C}_{10} \mathrm{H}_{20} \mathrm{O}_{2}$ ) fatty 

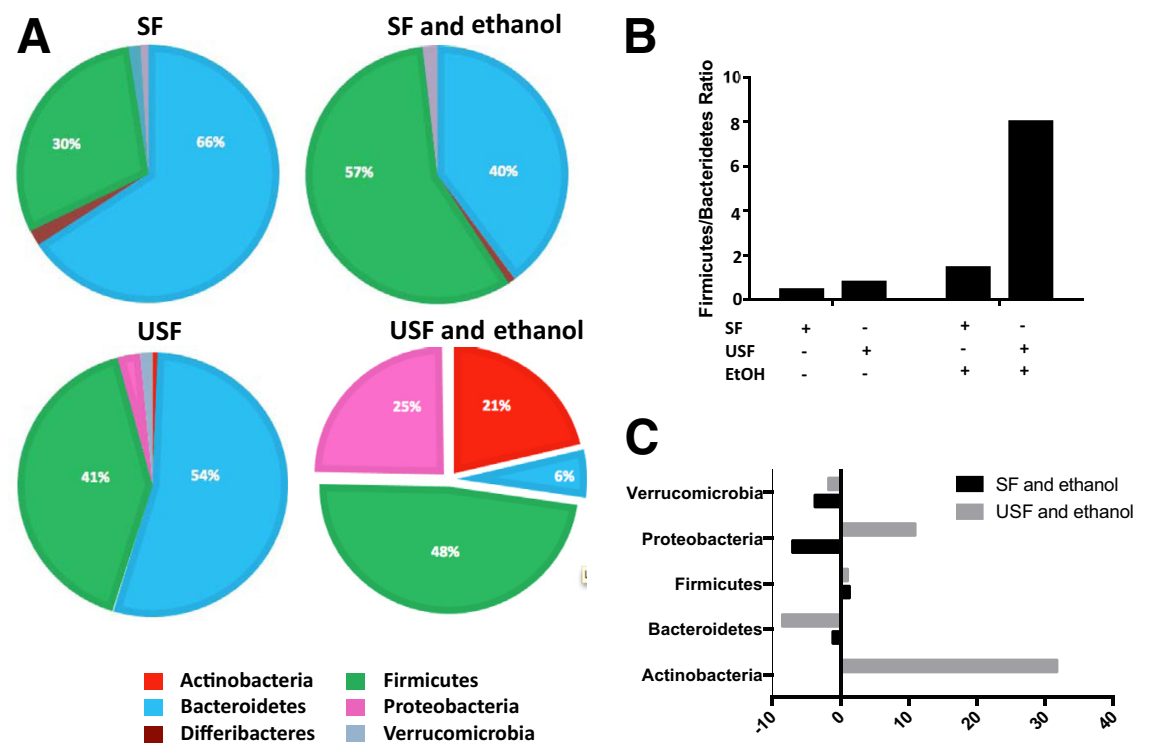

Figure 4 Effects of different types of dietary lipids and chronic ethanol (EtOH) administration on gut microbiota composition. A: Comparative analysis of the gut bacteria relative abundance in mice fed ethanol and different types of dietary lipids for 8 weeks. The phyla abundance is indicated by the color bars. Uncommon phyla that are a very small fraction of the total may not be visible in the chart even though they are present in the legend. B: Firmicutes:Bacteroidetes ratio at the end of the experiment. C: Changes in the gut bacteria phylum occur in response to 8 weeks of ethanol feeding compared with baseline. Results are presented as fold-changes over baseline set as $0 . \mathrm{SF}$, saturated fat; USF, unsaturated fat.

acids. The USF diet was enriched in corn oil; octadecadienoic (linoleic, $\mathrm{C}_{18} \mathrm{H}_{32} \mathrm{O}_{2}$ ) and octadecenoic (oleic, $\mathrm{C}_{18} \mathrm{H}_{34} \mathrm{O}_{2}$ ) fatty acids were the major fatty acids in this diet. At the end of experiment, we observed noticeable ethanol-induced changes in fecal metabolites in mice fed SF and USF diets compared with baseline and control animals fed SF or USF diets; significant differences in certain fecal metabolites were also seen between the SF and ethanol and USF and ethanol groups.

We examined the fecal metabolome changes that occurred in experimental animals in response to long-term ethanol exposure and different types of dietary fat feeding. Hence, we compared the metabolome status of mice fed SF or USF diets at baseline (T0, initiation of 5\% v/v ethanol feeding) and at the end of 8 weeks of alcohol administration (T8). Compared with baseline, the fecal metabolome changes associated with the ethanol exposure were characterized by a significant decrease in numerous amino acids in mice fed SF and USF diets, specifically, lysine, methionine, tyrosine, phenylalanine, and serine in the SF and ethanol group and serine and glycine in the USF and ethanol group (Table 1). There also were changes in fecal lipid metabolites at the end of 8 weeks of alcohol feeding (T8) compared with baseline (T0), including a moderate increase in hexadecanoic (palmitic, $\mathrm{C}_{16} \mathrm{H}_{32} \mathrm{O}_{2}$ ) acid that occurred regardless of the type of dietary fat and an increase in trans-9-octadecenoic (elaidic, transoleic, $\mathrm{C}_{18} \mathrm{H}_{34} \mathrm{O}_{2}$ ) and octadecadienoic (linoleic, $\mathrm{C}_{18} \mathrm{H}_{32} \mathrm{O}_{2}$ ) acids in the SF and ethanol and USF and ethanol groups, respectively. SF and ethanol feeding resulted in low abundance of several long chain fatty acids (LCFAs) (Table 2). The low levels of medium chain fatty acids (MCFAs) [octanoic (caprylic, $\mathrm{C}_{8} \mathrm{H}_{16} \mathrm{O}_{2}$ ) and hexanoic (caproic, $\mathrm{C}_{6} \mathrm{H}_{12} \mathrm{O}_{2}$ )] and short chain fatty acids (SCFAs) [pentanoic (valeric, $\mathrm{C}_{5} \mathrm{H}_{10} \mathrm{O}_{2}$ ), butanoic (butyric, $\mathrm{C}_{4} \mathrm{H}_{8} \mathrm{O}_{2}$ ), and propanoic (propionic, $\mathrm{C}_{3} \mathrm{H}_{6} \mathrm{O}_{2}$ )] were observed in USF and ethanol but not in $\mathrm{SF}$ and ethanol.

Next, we evaluated the effects of ethanol and different types of dietary lipids or their combination on the abundance of fecal metabolites at the end of the experiment (Table 3 and Figure 5, A-E). Importantly, diet had a major effect on the relative abundance of the observed changes in the fecal metabolites.

Table 1 Significant Changes of Fecal Amino Acids in Response to Ethanol and Dietary SF or USF

\begin{tabular}{|c|c|c|c|c|c|c|}
\hline Metabolite & Formula & CAS & ${ }^{1} t_{R}$ (seconds) & ${ }^{2} t_{R}$ (seconds) & Fold change* & $P$ value \\
\hline \multicolumn{7}{|c|}{ SF and ethanol (T8) vs baseline (T0) } \\
\hline L-Lysine & $\mathrm{C}_{6} \mathrm{H}_{14} \mathrm{~N}_{2} \mathrm{O}_{2}$ & $56-87-1$ & 2478.1 & 0.976 & -5.3 & 0.001 \\
\hline L-Methionine & $\mathrm{C}_{5} \mathrm{H}_{11} \mathrm{NO}_{2} \mathrm{~S}$ & $63-68-3$ & 2033.9 & 1.053 & -3.1 & 0.036 \\
\hline L-Tyrosine & $\mathrm{C}_{9} \mathrm{H}_{11} \mathrm{NO}_{3}$ & $60-18-4$ & 2747.8 & 1.281 & -3.1 & 0.026 \\
\hline L-Phenylalanine & $\mathrm{C}_{9} \mathrm{H}_{11} \mathrm{NO}_{2}$ & $63-91-2$ & 2174.0 & 1.095 & -2.4 & 0.046 \\
\hline L-Serine & $\mathrm{C}_{3} \mathrm{H}_{7} \mathrm{NO}_{3}$ & $56-45-1$ & 2054.0 & 0.930 & -2.5 & 0.004 \\
\hline \multicolumn{7}{|c|}{ USF and ethanol (T8) vs baseline (T0) } \\
\hline L-Serine ${ }^{\dagger}$ & $\mathrm{C}_{3} \mathrm{H}_{7} \mathrm{NO}_{3}$ & $56-45-1$ & 2054.0 & 0.930 & -3.8 & 0.007 \\
\hline L-Glycine & $\mathrm{C}_{2} \mathrm{H}_{5} \mathrm{NO}_{2}$ & $56-40-6$ & 1512.0 & 0.948 & -1.8 & 0.050 \\
\hline
\end{tabular}

*Results are presented as a ratio of the abundance between the fecal metabolites after 8 weeks of SF and ethanol or USF and ethanol feeding (T8) and baseline (T0). All molecules differ significantly $(P<0.05)$.

${ }^{\dagger} \mathrm{L}-$ Serine abundance differs significantly between mice fed SF and ethanol and those fed USF and ethanol at the end of 8 weeks of the experiment $(P<0.05)$.

CAS, chemical abstracts service; SF, saturated fat; $t_{R}$, retention time; USF, unsaturated fat. 
Table 2 Significant Changes of Fecal Lipid Metabolites in Response to Ethanol and Dietary SF or USF

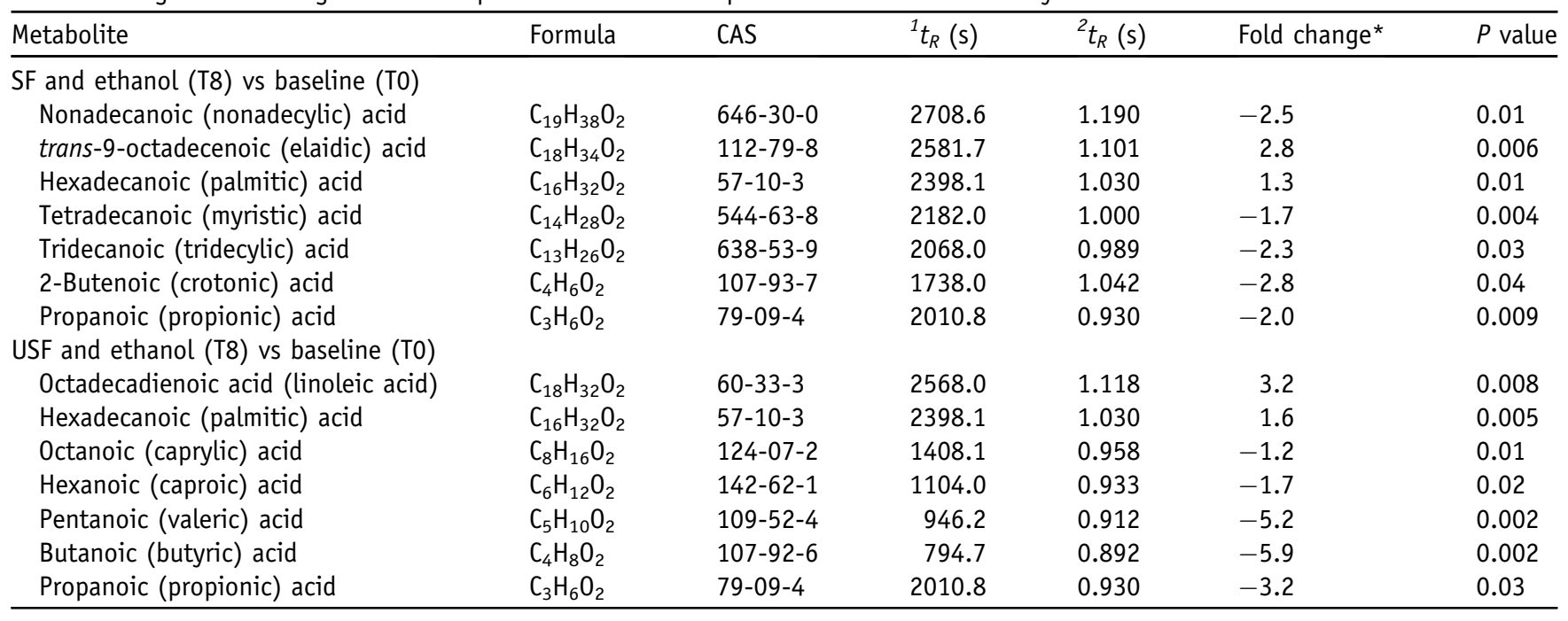

*Results are presented as a ratio of the abundance between the fecal metabolites after 8 weeks of SF and ethanol or USF and ethanol feeding (T8) and baseline (T0). All molecules differ significantly $(P<0.05)$.

CAS, chemical abstracts service; SF, saturated fat; $t_{R}$, retention time; USF, unsaturated fat.

After 8 weeks of feeding, dietary fat differentially altered the levels of numerous free fatty acids [eg, heptadecanoic (margaric, $\mathrm{C}_{17} \mathrm{H}_{34} \mathrm{O}_{2}$ ) and hexadecenoic (palmitoleic, $\mathrm{C}_{16} \mathrm{H}_{30} \mathrm{O}_{2}$ )] and the amino acid L-serine, with increased levels in animals fed SF compared with USF diets. When ethanol was added to the diets, a significant reduction in hexadecenoic (palmitoleic, $\mathrm{C}_{16} \mathrm{H}_{30} \mathrm{O}_{2}$ ) and octanoic (caprylic, $\mathrm{C} 8: 0$ ) acids was observed in animals fed USF diets; the levels of heptadecanoic (margaric, $\mathrm{C}_{17} \mathrm{H}_{34} \mathrm{O}_{2}$ ) acid were lower in mice fed SF and ethanol compared with mice fed SF diets. Notably, the levels of octanoic (caprylic, $\mathrm{C} 8: 0$ ), hexanoic (caproic, $\mathrm{C}_{6} \mathrm{H}_{12} \mathrm{O}_{2}$ ), and butanoic (butyric, $\mathrm{C}_{4} \mathrm{H}_{8} \mathrm{O}_{2}$ ) acids were elevated in response to $\mathrm{SF}$ and ethanol feeding compared with a SF diet alone, suggesting that lipid absorption most likely has not been impaired by ethanol administration. A recently published study also reported no differences in fat absorption between mice fed SF and ethanol compared with USF and ethanol. ${ }^{21}$ The fecal free fatty acids might be from the diet or may be products of microbial metabolism. Because animal fed the ethanol and control diet received the same amount of food (data not shown), the differences in certain free fatty acids between SF and ethanol versus SF and USF and ethanol versus USF are most likely due to the alterations of bacterial biosynthesis resulting from the gut microbiota dysbiosis ${ }^{21}$ and/or bacterial overgrowth that is frequently observed in ALD. ${ }^{14,17}$ There were significant differences in the abundance of several fecal free fatty acids between the mice fed SF and ethanol and USF and ethanol at the end of 8 weeks of

Table 3 Effects of Ethanol and Dietary Fat on the Abundance of Fecal Metabolites after 8 Weeks of Experimental Feeding

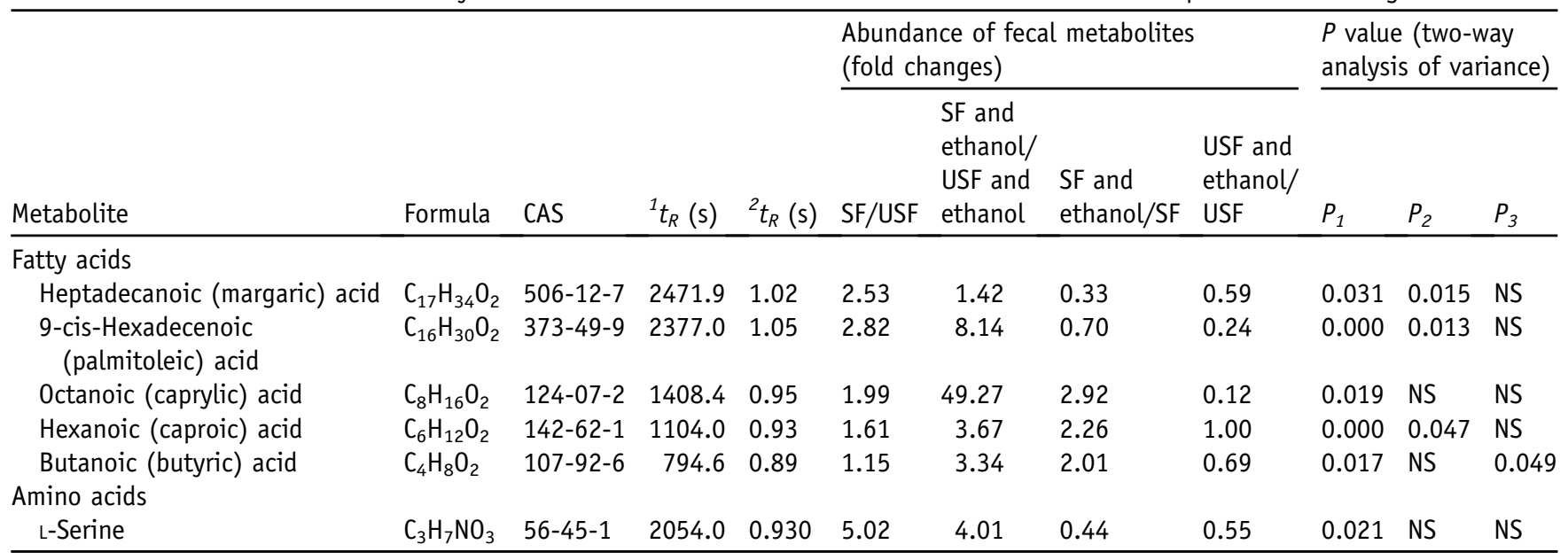

Results are presented as a ratio between the fecal metabolites of the specified groups after 8 weeks of feeding. $P_{1}$ is the $P$ value of fat factor, $P_{2}$ is the $P$ value of ethanol factor, and $P_{3}$ is the $P$ value of the synergistic effect of fat and ethanol factors.

CAS, chemical abstracts service; NS, not significant; SF, saturated fat; $t_{R}$, retention time; USF, unsaturated fat. 
A

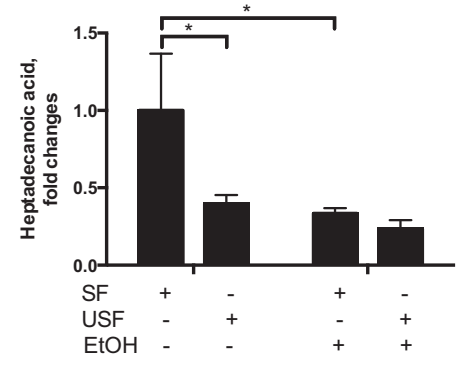

B

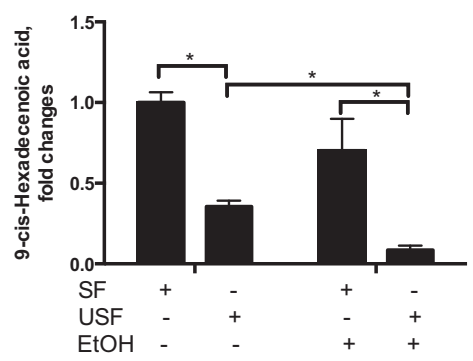

C

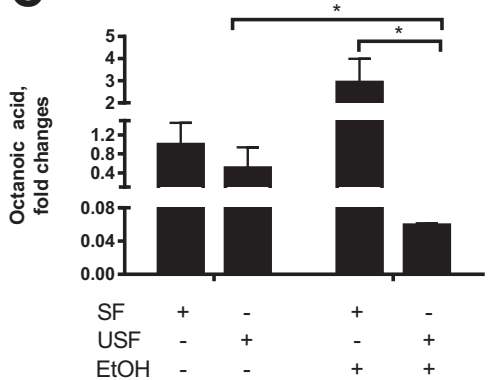

D

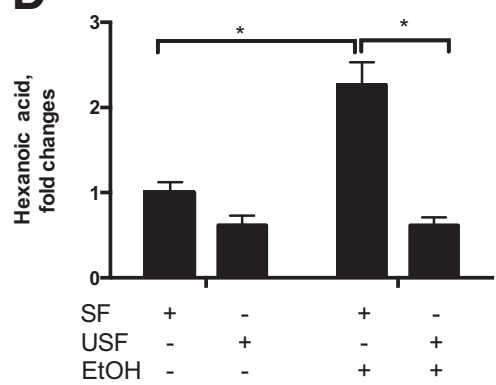

E

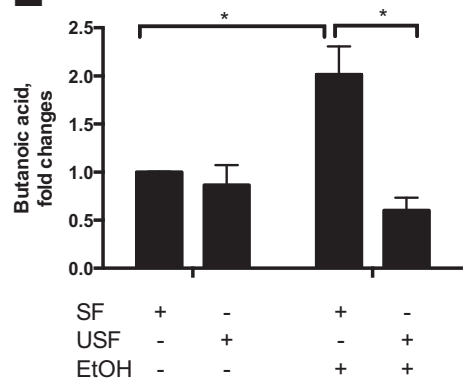

Figure 5 Effects of dietary fat, ethanol $(\mathrm{EtOH})$, and their combination on changes of fecal free fatty acids after 8 weeks of experiment. A: Heptadecanoic (margaric, $\mathrm{C}_{17} \mathrm{H}_{34} \mathrm{O}_{2}$ ) acid. B: 9-cis-Hexadecenoic (palmitoleic, $\mathrm{C}_{16} \mathrm{H}_{30} \mathrm{O}_{2}$ ) acid. C: Octanoic (caprylic, $\mathrm{C}_{8} \mathrm{H}_{16} \mathrm{O}_{2}$ ) acid. D: Hexanoic (caproic, $\mathrm{C}_{6} \mathrm{H}_{12} \mathrm{O}_{2}$ ) acid. E: Butanoic (butyric, $\mathrm{C}_{4} \mathrm{H}_{8} \mathrm{O}_{2}$ ) acid. Results are presented as fold-changes over the saturated fat (SF) set as $1 .{ }^{*} P<0.05$. USF, unsaturated fat.

experimental feeding. Among LCFAs, hexadecenoic (palmitoleic, $\mathrm{C}_{16} \mathrm{H}_{30} \mathrm{O}_{2}$ ) acid was significantly decreased in the USF and ethanol group compared with the SF and ethanol group. Long-term USF and ethanol administration resulted in significantly decreased levels of fecal MCFAs, including hexanoic (caproic, C6:0) and octanoic (caprylic, C8:0) acids. Remarkably, the levels of octanoic (caprylic, C8:0) acid (known to have some antimicrobial proper$\operatorname{ties}^{41}$ ) were markedly reduced (approximately 48 -fold) in mice fed USF and ethanol compared with mice fed SF and ethanol. Another important observation is that the levels of butanoic (butyric, $\mathrm{C}_{4} \mathrm{H}_{8} \mathrm{O}_{2}$ ) acid were significantly lower in mice fed USF and ethanol compared with mice fed SF and ethanol, with a significant interaction between a diet and ethanol (two-way analysis of variance).

\section{Discussion}

Dietary fat and alcohol play important roles in the pathogenesis of ALD. Indeed, the alcohol-nutrient interactions may help to explain why only some individuals who drink heavily develop ALD. The protective effects of dietary SF and deleterious effects of dietary USF, primarily linoleic acid from corn oil, on ethanol-induced liver injury and intestinal barrier disruption are increasingly recognized and well documented in experimental animal models of ALD. ${ }^{11,21,32,42-44}$ Moreover, an epidemiologic study that analyzed cirrhosis mortality data in various countries with similar per capita alcohol consumption has reported that dietary intake of SF is associated with lower mortality rates, whereas dietary intake of USF is associated with a higher mortality associated with alcoholic cirrhosis. ${ }^{45}$ However, the underlying mechanism(s) by which different types of dietary fat potentiate or attenuate ALD is not completely understood. Crosstalk between the gut and liver is an important determinant of alcohol-induced liver disease. ${ }^{46}$ Recent studies have implicated the alterations of gut microbiota in ALD development and progression. ${ }^{12,19-21}$ The gastrointestinal microbiome is composed of trillions of organisms, ${ }^{47}$ which perform a diverse range of metabolic functions, ${ }^{48}$ including production of numerous metabolites that serve as the nutritional sources for microbes and important messengers between the microbiota and the host. ${ }^{49}$ Therefore, the host-microbe metabolic homeostasis is essential for the host health. The focus of the current study was to evaluate the changes in the gut microbiome and metabolome that occurred in response to varying dietary fat in combination with ethanol and to evaluate how these changes affect endotoxemia and liver health in an animal model of ALD.

The most significant finding of the current study is that compared with a USF diet rich in corn oil, the diet rich in SF (mainly MCTs) prevented ethanol-induced changes in the gut microbiota and associated liver injury. Specifically, USF and ethanol-mediated reduction of Bacteroidetes and increases in Proteobacteria and Actinobacteria phyla did not occur in response to SF and ethanol feeding. Importantly, mice fed USF and ethanol developed profound liver steatosis and injury, whereas mice fed SF and ethanol were protected against ALD. Similar to our animal findings, recently published human studies reported low abundances of Bacteroidetes and higher 
Proteobacteria in alcoholics with dysbiosis ${ }^{38}$ and in patients with cirrhosis (both hepatitis B and alcohol related). ${ }^{50}$ In contrast, an ethanol-mediated increase in Bacteroidetes has been observed in experimental rodent ALD ${ }^{20,21}$ However, it is difficult to compare results from different experimental studies directly because the discrepancies might be due to several factors, including different animal models of ALD (eg, ad libitum versus intragastric ethanol administration), different durations of alcohol feeding, and variability in the specific dietary components (eg, diets containing different types of fat and/or fermentable fibers). The alterations in the gut microbiota community observed in our study were associated with liver inflammation and injury, suggesting that USF and ethanol-mediated expansion of the Proteobacteria and Actinobacteria phyla plays a pathogenic role in the development of ALD. The exact mechanism(s) underlying these effects remain to be determined.

Alcohol-induced alterations of the gut microbiota are linked with endotoxemia and increased gut permeability. ${ }^{7,12,19,21,38}$ This might be one potential mechanism of how impaired gut microbiota contribute to ALD. It is well known that ethanolinduced endotoxemia results in activation of hepatic resident (Kupffer cells) and infiltrating macrophages, leading to overproduction of proinflammatory cytokines and subsequent liver inflammation and injury. In the context of this study, the increase in Proteobacteria phylum (gram-negative bacteria) provides a possible link between the alterations of the gut microbiota and hepatic inflammation via endotoxin (LPS), a component of the gram-negative bacteria outer membrane. It is noteworthy that unlike dietary SF, USF feeding promoted ethanol-mediated reduction of commensal bacteria (eg, Lactobacillus species) that produce beneficial factors for maintaining barrier function in intestinal epithelial cells. ${ }^{21} \mathrm{An}$ increase in gram-negative Akkermansia muciniphila, a bacteria involved in maintaining the intestinal mucous layer integrity, has been found in experimental ALD. ${ }^{20,24}$ We have recently reported that Corynebacterium and Alcaligenes were the most significantly overgrown bacterial genera in response to alcohol feeding among the Proteobacteria and Actinobacteria phyla, respectively. ${ }^{19}$ Although, alcohol-mediated changes in the gut microbiota have been extensively studied in experimental and human ALD, their causal role in the development and progression of ALD is poorly understood. Inoculation of germfree animals with single or groups of bacteria may help to determine the possible causal role of specific bacteria in alcohol-induced liver injury.

In the present study, the differences in the microbial phyla between mice fed SF and ethanol compared with those fed USF and ethanol were associated with the noticeable changes in the fecal metabolites, including a decrease in several LCFAs, MCFAs, and SCFAs. It has been demonstrated that the low levels of microbial LCFAs may compromise the growth of Lactobacillus, ${ }^{21}$ a bacteria with known beneficial effects in ALD, via multiple mechanisms, including maintaining gut barrier integrity. ${ }^{12,21,27,28,51}$ In our study, the most prominent alteration (approximately 50-fold decrease in
USF and ethanol versus SF and ethanol) was observed in the abundance of octanoic acid, which possesses some antibacterial properties. ${ }^{41,52}$ Therefore, the deficiency of octanoic acid most likely contributes to the expansion of certain types of bacteria and overall changes in the gut microbial population caused by USF and ethanol. The protective effects of the SF diet on ethanol-mediated changes of the gut microbiota might be also attributed to octanoic acid because it was the major fatty acid in the SF diet. Further studies will be needed to test these hypotheses.

Through the process of fermentation, colonic bacteria are able to produce SCFAs, with acetate, propionate, and butyrate being the major SCFAs in the mammalian gut. ${ }^{53}$ The type and amount of produced SCFAs depend on numerous factors, including diet, intestinal transit, and microbiota composition. ${ }^{54}$ In the present study, low SCFA levels (butyrate specifically) were found in mice fed USF and ethanol compared with those fed SF and ethanol, even though both groups received a similar amount of carbohydrate and proteins. Thus, the low SCFA levels were likely due to the marked changes in the gut microbiota composition induced by the ethanol and USF diet. Given that SCFAs play an important role in intestinal and host health,$^{55}$ the decrease in fecal SCFAs may contribute to the intestinal barrier disruption observed in mice fed USF and ethanol. Low SCFA levels may compromise mucosal immune tolerance ${ }^{56,57}$ and intestinal epithelia energy supply because normal epithelia derive $60 \%$ to $70 \%$ of their energy from SCFAs, particularly butyrate. ${ }^{58}$ In addition, butyrate possesses anti-inflammatory properties in part via inhibition of NF- $\kappa \mathrm{B}$ in human colonic epithelial cells $s^{59}$ and may serve as a potent histone deacetylase inhibitor. ${ }^{60}$ In experimental ALD, butyrate supplementation protected against alcohol-mediated intestinal tight junction disruption and liver inflammation. ${ }^{61}$ SCFAs produced by the gut microbiota can be found in hepatic, portal, and peripheral blood. ${ }^{62}$ These SCFAs affect lipid, glucose, and cholesterol metabolism in various tissues, including liver, by reducing plasma and hepatic fatty acid content, lowering cholesterol, and potentially improving insulin sensitivity. ${ }^{63-68}$ Further investigations into the mechanisms by which ethanol alters bacterial fermentation and SCFA production and how these metabolites affect the intestine and the liver are necessary. Alcohol-mediated alterations in both gut microbiota composition and functionality in association with the increased gut permeability have been recently reported in humans. Compared with control individuals, the fecal metabolome of alcohol-dependent individuals was characterized by the presence or absence of certain compounds (eg, alcohols, alkanes, and benzenes or 2-methyl-1-butanol and methanethiol). Another comprehensive fecal metabolome analysis in humans has revealed numerous alcohol-induced alterations, including a decrease in propionate, isobutyrate, and camphene, a natural product and hepatic steatosis attenuator, among others. ${ }^{69}$

The data obtained in our study contribute to the increasing body of evidence suggesting that the alterations of the hostmicrobiome and nutrient-microbiome interactions are critically involved in the pathogenesis of numerous diseases, including 
ALD. Our findings support the concept that alterations in the gut microbial community and metabolic activity are important factors in ALD pathogenesis. Importantly, the findings from this study indicate that the ethanol-mediated gut dysbiosis can be therapeutically targeted by dietary manipulations (eg, modulation of dietary lipids) that may offer a novel prevention and therapeutic approach to ALD management. The study has several strengths, such as simultaneous metagenomic and metabolomic analysis that identifies novel mechanisms underlying the complex interaction of the gut microbiota and the host and the evaluation of the gut-liver interactions in response to different types of dietary fat that allows us to better understand the role of nutrition in ALD development and progression. A limitation of the study is that the gut microbial community has been examined in the fecal samples. Although the stool microbiota (intestinal lumen microbiota) may have no direct contact with the epithelial cells, they can act through cometabolism or metabolic exchange with the host. Still, the role of the adherent mucosa-associated microbiota that most likely affect intestinal epithelial cell function and intestinal barrier integrity needs to be further investigated.

To summarize, long-term alcohol consumption is accompanied by alterations in gut microbiota composition and metabolic potential, and dietary factors likely play a critical role in shaping these changes. However, the exact mechanism(s) and the contributions of ethanol, products of ethanol metabolism, specific dietary nutrients, and their combination to the gut dysbiosis and/or alterations of microbial metabolic activity need to be further defined. The molecular mechanism(s) by which ethanol modulates microbial metabolic activity also remains to be established. Therefore, further research is required on the interactions among the specific types of dietary lipids, gut microbiota community structure, metabolic function, and ALD development and progression.

\section{Acknowledgments}

We thank Jingwen Zhang for assisting with tissue staining and Marion McClain for proofreading the manuscript.

\section{References}

1. McClain CM, Carithers RL: Alcoholic liver disease. Edited by Feldman M, Friedman L, Brandt L. Sleisenger and Fordtran's Gastrointestinal and Liver Disease Pathophysiology/Diagnosis/ Management. ed 8. Canada, Saunders Elsevier, 2006, pp 1771-1792

2. D'Amico G, Garcia-Tsao G, Pagliaro L: Natural history and prognostic indicators of survival in cirrhosis: a systematic review of 118 studies. J Hepatol 2006, 44:217-231

3. Wakim-Fleming J, Mullen KD: Long-term management of alcoholic liver disease. Clin Liver Dis 2005, 9:135-149

4. Szabo G, Bala S: Alcoholic liver disease and the gut-liver axis. World J Gastroenterol 2010, 16:1321-1329

5. Wang HJ, Zakhari S, Jung MK: Alcohol, inflammation, and gut-liverbrain interactions in tissue damage and disease development. World J Gastroenterol 2010, 16:1304-1313

6. Purohit V, Bode JC, Bode C, Brenner DA, Choudhry MA, Hamilton F, Kang YJ, Keshavarzian A, Rao R, Sartor RB,
Swanson C, Turner JR: Alcohol, intestinal bacterial growth, intestinal permeability to endotoxin, and medical consequences: summary of a symposium. Alcohol 2008, 42:349-361

7. Leclercq S, Matamoros S, Cani PD, Neyrinck AM, Jamar F, Starkel P, Windey K, Tremaroli V, Backhed F, Verbeke K, de Timary P, Delzenne NM: Intestinal permeability, gut-bacterial dysbiosis, and behavioral markers of alcohol-dependence severity. Proc Natl Acad Sci U S A 2014, 111:E4485-E4493

8. Bala S, Marcos M, Gattu A, Catalano D, Szabo G: Acute binge drinking increases serum endotoxin and bacterial DNA levels in healthy individuals. PLoS One 2014, 9:e96864

9. Fukui H, Brauner B, Bode JC, Bode C: Plasma endotoxin concentrations in patients with alcoholic and non-alcoholic liver disease: reevaluation with an improved chromogenic assay. J Hepatol 1991, 12:162-169

10. Parlesak A, Schafer C, Schutz T, Bode JC, Bode C: Increased intestinal permeability to macromolecules and endotoxemia in patients with chronic alcohol abuse in different stages of alcohol-induced liver disease. J Hepatol 2000, 32:742-747

11. Kirpich IA, Feng W, Wang Y, Liu Y, Barker DF, Barve SS, McClain CJ: The type of dietary fat modulates intestinal tight junction integrity, gut permeability, and hepatic toll-like receptor expression in a mouse model of alcoholic liver disease. Alcohol Clin Exp Res 2012, $36: 835-846$

12. Mutlu E, Keshavarzian A, Engen P, Forsyth CB, Sikaroodi M, Gillevet P: Intestinal dysbiosis: a possible mechanism of alcoholinduced endotoxemia and alcoholic steatohepatitis in rats. Alcohol Clin Exp Res 2009, 33:1836-1846

13. Keshavarzian A, Farhadi A, Forsyth CB, Rangan J, Jakate S, Shaikh M, Banan A, Fields JZ: Evidence that chronic alcohol exposure promotes intestinal oxidative stress, intestinal hyperpermeability and endotoxemia prior to development of alcoholic steatohepatitis in rats. J Hepatol 2009, 50:538-547

14. Gabbard SL, Lacy BE, Levine GM, Crowell MD: The impact of alcohol consumption and cholecystectomy on small intestinal bacterial overgrowth. Dig Dis Sci 2014, 59:638-644

15. Kirpich IA, Solovieva NV, Leikhter SN, Shidakova NA, Lebedeva OV, Sidorov PI, Bazhukova TA, Soloviev AG, Barve SS, McClain CJ, Cave M: Probiotics restore bowel flora and improve liver enzymes in human alcohol-induced liver injury: a pilot study. Alcohol 2008, 42:675-682

16. Bode C, Kolepke R, Schafer K, Bode JC: Breath hydrogen excretion in patients with alcoholic liver disease-evidence of small intestinal bacterial overgrowth. Z Gastroenterol 1993, 31:3-7

17. Morencos FC, de las Heras Castano G, Martin Ramos L, Lopez Arias MJ, Ledesma F, Pons Romero F: Small bowel bacterial overgrowth in patients with alcoholic cirrhosis. Dig Dis Sci 1995, 40: $1252-1256$

18. Tuomisto S, Pessi T, Collin P, Vuento R, Aittoniemi J, Karhunen PJ: Changes in gut bacterial populations and their translocation into liver and ascites in alcoholic liver cirrhotics. BMC Gastroenterol 2014, 14:40

19. Bull-Otterson L, Feng W, Kirpich I, Wang Y, Qin X, Liu Y, Gobejishvili L, Joshi-Barve S, Ayvaz T, Petrosino J, Kong M, Barker D, McClain C, Barve S: Metagenomic analyses of alcohol induced pathogenic alterations in the intestinal microbiome and the effect of Lactobacillus rhamnosus GG treatment. PLoS One 2013 , 8:e53028

20. Yan AW, Fouts DE, Brandl J, Starkel P, Torralba M, Schott E, Tsukamoto H, Nelson KE, Brenner DA, Schnabl B: Enteric dysbiosis associated with a mouse model of alcoholic liver disease. Hepatology 2011, 53:96-105

21. Chen P, Torralba M, Tan J, Embree M, Zengler K, Starkel P, van Pijkeren JP, DePew J, Loomba R, Ho SB, Bajaj JS, Mutlu EA, Keshavarzian A, Tsukamoto H, Nelson KE, Fouts DE, Schnabl B: Supplementation of saturated long-chain fatty acids maintains intestinal eubiosis and reduces ethanol-induced liver injury in mice. Gastroenterology 2015, 148:203-214.e16 
22. Baraona E, Julkunen R, Tannenbaum L, Lieber CS: Role of intestinal bacterial overgrowth in ethanol production and metabolism in rats. Gastroenterology 1986, 90:103-110

23. Basuroy S, Sheth P, Mansbach CM, Rao RK: Acetaldehyde disrupts tight junctions and adherens junctions in human colonic mucosa: protection by EGF and L-glutamine. Am J Physiol Gastrointest Liver Physiol 2005, 289:G367-G375

24. Hartmann P, Chen P, Wang HJ, Wang L, McCole DF, Brandl K, Starkel P, Belzer C, Hellerbrand C, Tsukamoto H, Ho SB, Schnabl B: Deficiency of intestinal mucin-2 ameliorates experimental alcoholic liver disease in mice. Hepatology 2013, 58:108-119

25. Ferrier L, Berard F, Debrauwer L, Chabo C, Langella P, Bueno L, Fioramonti J: Impairment of the intestinal barrier by ethanol involves enteric microflora and mast cell activation in rodents. Am J Pathol 2006, 168:1148-1154

26. Adachi Y, Moore LE, Bradford BU, Gao W, Thurman RG: Antibiotics prevent liver injury in rats following long-term exposure to ethanol. Gastroenterology 1995, 108:218-224

27. Forsyth CB, Farhadi A, Jakate SM, Tang Y, Shaikh M, Keshavarzian A: Lactobacillus GG treatment ameliorates alcoholinduced intestinal oxidative stress, gut leakiness, and liver injury in a rat model of alcoholic steatohepatitis. Alcohol 2009, 43: $163-172$

28. Wang Y, Kirpich I, Liu Y, Ma Z, Barve S, McClain CJ, Feng W: Lactobacillus rhamnosus GG treatment potentiates intestinal hypoxiainducible factor, promotes intestinal integrity and ameliorates alcoholinduced liver injury. Am J Pathol 2011, 179:2866-2875

29. Nanji AA, French SW: Dietary linoleic acid is required for development of experimentally induced alcoholic liver injury. Life Sci 1989, 44:223-227

30. Nanji AA, Jokelainen K, Tipoe GL, Rahemtulla A, Dannenberg AJ: Dietary saturated fatty acids reverse inflammatory and fibrotic changes in rat liver despite continued ethanol administration. J Pharmacol Exp Ther 2001, 299:638-644

31. Nanji AA, Yang EK, Fogt F, Sadrzadeh SM, Dannenberg AJ: Medium chain triglycerides and vitamin E reduce the severity of established experimental alcoholic liver disease. J Pharmacol Exp Ther 1996, 277:1694-1700

32. Kirpich IA, Feng W, Wang Y, Liu Y, Beier JI, Arteel GE, Falkner KC, Barve SS, McClain CJ: Ethanol and dietary unsaturated fat (corn oil/linoleic acid enriched) cause intestinal inflammation and impaired intestinal barrier defense in mice chronically fed alcohol. Alcohol 2013, 47:257-264

33. Kirpich IA, Gobejishvili LN, Bon Homme M, Waigel S, Cave M, Arteel G, Barve SS, McClain CJ, Deaciuc IV: Integrated hepatic transcriptome and proteome analysis of mice with high-fat dietinduced nonalcoholic fatty liver disease. J Nutr Biochem 2011, 22 : $38-45$

34. HMP-Consortium: Evaluation of $16 \mathrm{~S}$ rDNA-based community profiling for human microbiome research. PLoS One 2012, 7: e39315

35. Maidak BL, Olsen GJ, Larsen N, Overbeek R, McCaughey MJ, Woese CR: The RDP (Ribosomal Database Project). Nucleic Acids Res 1997, 25:109-111

36. Wei X, Shi X, Koo I, Kim S, Schmidt RH, Arteel GE, Watson WH, McClain C, Zhang X: MetPP: a computational platform for comprehensive two-dimensional gas chromatography time-of-flight mass spectrometry-based metabolomics. Bioinformatics 2013, 29: $1786-1792$

37. Zhang J, Fang A, Wang B, Kim SH, Bogdanov B, Zhou Z, McClain C, Zhang X: iMatch: a retention index tool for analysis of gas chromatography-mass spectrometry data. J Chromatogr A 2011, 1218:6522-6530

38. Mutlu EA, Gillevet PM, Rangwala H, Sikaroodi M, Naqvi A, Engen PA, Kwasny M, Lau CK, Keshavarzian A: Colonic microbiome is altered in alcoholism. Am J Physiol Gastrointest Liver Physiol 2012, 302:G966-G978
39. Ley RE, Turnbaugh PJ, Klein S, Gordon JI: Microbial ecology: human gut microbes associated with obesity. Nature 2006, 444: $1022-1023$

40. Chen P, Starkel P, Turner JR, Ho SB, Schnabl B: Dysbiosis-induced intestinal inflammation activates tumor necrosis factor receptor I and mediates alcoholic liver disease in mice. Hepatology 2015, 61: 883-894

41. Valipe SR, Nadeau JA, Annamali T, Venkitanarayanan K, Hoagland T: In vitro antimicrobial properties of caprylic acid, monocaprylin, and sodium caprylate against Dermatophilus congolensis. Am J Vet Res 2011, 72:331-335

42. Ronis MJ, Korourian S, Zipperman M, Hakkak R, Badger TM: Dietary saturated fat reduces alcoholic hepatotoxicity in rats by altering fatty acid metabolism and membrane composition. J Nutr 2004, 134: 904-912

43. Zhong W, Li Q, Xie G, Sun X, Tan X, Sun X, Jia W, Zhou Z: Dietary fat sources differentially modulate intestinal barrier and hepatic inflammation in alcohol-induced liver injury in rats. Am J Physiol Gastrointest Liver Physiol 2013, 305:G919-G932

44. Nanji AA: Role of different dietary fatty acids in the pathogenesis of experimental alcoholic liver disease. Alcohol 2004, 34:21-25

45. Nanji AA, French SW: Dietary factors and alcoholic cirrhosis. Alcohol Clin Exp Res 1986, 10:271-273

46. Szabo G: Gut-liver axis in alcoholic liver disease. Gastroenterology 2015, 148:30-36

47. Whitman WB, Coleman DC, Wiebe WJ: Prokaryotes: the unseen majority. Proc Natl Acad Sci U S A 1998, 95:6578-6583

48. Tremaroli V, Backhed F: Functional interactions between the gut microbiota and host metabolism. Nature 2012, 489:242-249

49. Nicholson JK, Holmes E, Kinross J, Burcelin R, Gibson G, Jia W, Pettersson S: Host-gut microbiota metabolic interactions. Science 2012, 336:1262-1267

50. Chen Y, Yang F, Lu H, Wang B, Chen Y, Lei D, Wang Y, Zhu B, Li L: Characterization of fecal microbial communities in patients with liver cirrhosis. Hepatology 2011, 54:562-572

51. Wang Y, Liu Y, Kirpich I, Ma Z, Wang C, Zhang M, Suttles J, McClain C, Feng W: Lactobacillus rhamnosus GG reduces hepatic TNFalpha production and inflammation in chronic alcohol-induced liver injury. J Nutr Biochem 2013, 24:1609-1615

52. Zheng CJ, Yoo JS, Lee TG, Cho HY, Kim YH, Kim WG: Fatty acid synthesis is a target for antibacterial activity of unsaturated fatty acids. FEBS Lett 2005, 579:5157-5162

53. Wong JM, de Souza R, Kendall CW, Emam A, Jenkins DJ: Colonic health: fermentation and short chain fatty acids. J Clin Gastroenterol 2006, 40:235-243

54. Macfarlane GT, Macfarlane S: Bacteria, colonic fermentation, and gastrointestinal health. J AOAC Int 2012, 95:50-60

55. Topping DL, Clifton PM: Short-chain fatty acids and human colonic function: roles of resistant starch and nonstarch polysaccharides. Physiol Rev 2001, 81:1031-1064

56. Maslowski KM, Vieira AT, Ng A, Kranich J, Sierro F, Yu D, Schilter HC, Rolph MS, Mackay F, Artis D, Xavier RJ, Teixeira MM, Mackay CR: Regulation of inflammatory responses by gut microbiota and chemoattractant receptor GPR43. Nature 2009, 461:1282-1286

57. Kim MH, Kang SG, Park JH, Yanagisawa M, Kim CH: Short-chain fatty acids activate GPR41 and GPR43 on intestinal epithelial cells to promote inflammatory responses in mice. Gastroenterology 2013, 145:396-406.e1-10

58. Scheppach W: Effects of short chain fatty acids on gut morphology and function. Gut 1994, 35:S35-S38

59. Inan MS, Rasoulpour RJ, Yin L, Hubbard AK, Rosenberg DW, Giardina C: The luminal short-chain fatty acid butyrate modulates NF-kappaB activity in a human colonic epithelial cell line. Gastroenterology 2000, 118:724-734

60. Davie JR: Inhibition of histone deacetylase activity by butyrate. J Nutr 2003, 133:2485S-2493S 
61. Cresci GA, Bush K, Nagy LE: Tributyrin supplementation protects mice from acute ethanol-induced gut injury. Alcohol Clin Exp Res 2014, 38:1489-1501

62. Cummings JH, Pomare EW, Branch WJ, Naylor CP, Macfarlane GT: Short chain fatty acids in human large intestine, portal, hepatic and venous blood. Gut 1987, 28:1221-1227

63. Kimura I, Ozawa K, Inoue D, Imamura T, Kimura K, Maeda T, Terasawa K, Kashihara D, Hirano K, Tani T, Takahashi T, Miyauchi S, Shioi G, Inoue H, Tsujimoto G: The gut microbiota suppresses insulinmediated fat accumulation via the short-chain fatty acid receptor GPR43. Nat Commun 2013, 4:1829

64. Yamashita H, Fujisawa K, Ito E, Idei S, Kawaguchi N, Kimoto M, Hiemori M, Tsuji H: Improvement of obesity and glucose tolerance by acetate in Type 2 diabetic Otsuka Long-Evans Tokushima Fatty (OLETF) rats. Biosci Biotechnol Biochem 2007, 71: 1236-1243
65. Lin HV, Frassetto A, Kowalik EJ Jr, Nawrocki AR, Lu MM, Kosinski JR, Hubert JA, Szeto D, Yao X, Forrest G, Marsh DJ: Butyrate and propionate protect against diet-induced obesity and regulate gut hormones via free fatty acid receptor 3-independent mechanisms. PLoS One 2012, 7:e35240

66. Kondo T, Kishi M, Fushimi T, Kaga T: Acetic acid upregulates the expression of genes for fatty acid oxidation enzymes in liver to suppress body fat accumulation. J Agric Food Chem 2009, 57:5982-5986

67. Wright RS, Anderson JW, Bridges SR: Propionate inhibits hepatocyte lipid synthesis. Proc Soc Exp Biol Med 1990, 195:26-29

68. Hara H, Haga S, Aoyama Y, Kiriyama S: Short-chain fatty acids suppress cholesterol synthesis in rat liver and intestine. J Nutr 1999 , 129:942-948

69. Couch RD, Dailey A, Zaidi F, Navarro K, Forsyth CB, Mutlu E, Engen PA, Keshavarzian A: Alcohol induced alterations to the human fecal VOC metabolome. PLoS One 2015, 10:e0119362 